\title{
Toripalimab-Induced Dermatomyositis in a Patient with Metastatic Melanoma
}

Jingrong Lin · Minmin Xue · Mingyang Gao • Pu Yu • Shixin Han

\section{ABSTRACT}

Toripalimab is a monoclonal antibody targeting programmed cell death protein 1 (PD-1). It has recently been approved as an immune checkpoint inhibitor in second-line therapies in patients with unresectable or metastatic melanoma; however, it may be associated with various immune-related adverse events (irAEs). Here we report a case of toripalimab-induced dermatomyositis in a patient receiving treatment for metastatic melanoma. The symptoms were relieved by discontinuing toripalimab and administering once-daily intravenous methylprednisolone $1 \mathrm{mg} / \mathrm{kg}$. We suggest that this case serves a warning to clinicians of the need to be aware of the possiblilty of toripalimab-induced dermatomyositis. Early recognition and treatment may prevent progression and improve prognosis of this irAE.

Keywords: Dermatomyositis; irAEs; Metastatic melanoma; Toripalimab; Treatment

Digital Features To view digital features for this article go to https://doi.org/10.6084/m9.figshare.12279923.

J. Lin $\cdot$ M. Xue $\cdot$ M. Gao $\cdot$ P. Yu $\cdot$ S. Han $(\bowtie)$

Department of Dermatology, First Affiliated

Hospital of Dalian Medical University, Dalian, China

e-mail: hanshixin@126.com 


\section{Key Summary Points}

A 66-year-old man with history of malignant melanoma presented with pain and weakness in the upper limbs; dysphagia; heliotrope rash on the head, face, and chest; and purplish-red scaly maculopapules and poikiloderma on the scapular region, upper arm extension, and dorsal hands, with occasional pruritus. These symptoms developed after 2 cycles of immunotherapy with toripalimab, a newly approved inhibitor of programmed cell death protein 1 .

Laboratory evaluations indicated elevated levels of creatine kinase (654 U/L), cretine kinase-MB $(6.11 \mu \mathrm{g} / \mathrm{L})$, and lactic dehydrogenase (259 U/L), and positive antinuclear antibodies (titier of 1:100), with a speckled pattern.

Electroneuromyography revealed myogenic involvement of the deltoid muscle.

The patient was diagnosed with dermatomyositis. Treatment with toripalimab was discontinued, and the dermatomyositis was successfully treated with methylprednisolone at a once-daily dose of $1 \mathrm{mg} / \mathrm{kg}$ body weight.

This is the first case report of dermatomyositis as an immune-related adverse event induced by toripalimab. It should serve as a warning to clinicians that they need to be aware of the potential for immunotherapy-induced dermatomyositis and prompt early recognition of the entity.

\section{INTRODUCTION}

Toripalimab is a monoclonal antibody targeting the programmed cell death protein 1 (PD-1). It was approved in December 2018 by China's
National Medical Products Authority (NMPA) as an immune checkpoint inhibitor in second-line treatment in patients with unresectable or metastatic melanoma following failure of the first-line treatment. As a checkpoint inhibitor, this drug may be associated with various immune-related adverse events (irAEs), including fatigue, dermatitis, colitis, hepatitis, and dermatomyositis [1]. Here, we report the first case of toripalimab-induced dermatomyositis in a patient with metastatic melanoma, which was successfully treated with intravenous corticosteroid and discontinuation of toripalimab therapy. Informed consent was obtained from the patient for publication of the article. We thank the patient for his written consent for using his pictures and data.

\section{CASE PRESENTATION}

A 66-year-old man without a significant medical history presented with a histopathologically diagnosed malignant melanoma on the left leg. He had undergone wide local resection 2 years previously. Immunohistochemistry studies showed positivity for Melan-A, HMB45, S100, and Ki-67 (60-70\%). One year earlier, he had complained of an enlarged mass in the left inguinal region. Fine needle aspiration of the enlarged mass revealed it to be metastatic melanoma, with DDR2 amplification, IL7R amplification, NOTCH2 amplification, DNM3-EPHA2 fusion, NRAS amplification with a G12D mutation, MAXS141N mutation, PDK1G382R mutation, and QKIL178V mutation. Ten months before his current presentation at our hospital, he had been started on treatment with intravenous toripalimab $240 \mathrm{mg}$ every 2 weeks. One month after the initiation of the toripalimab treatment, he developed pain and weakness in the upper limbs; dysphagia; heliotrope rash on the head, face, and chest; purplish-red scaly maculopapules; and poikiloderma on the scapular region, upper arm extension, and dorsal hands, with occasional pruritus (Fig. 1). Laboratory evaluations indicated elevated levels of creatine kinase $(654 \mathrm{U} / \mathrm{L})$, cretine kinase-MB $(6.11 \mu \mathrm{g} / \mathrm{L})$, and lactic dehydrogenase $(259 \mathrm{U} / \mathrm{L})$. Testing for antinuclear antibodies was positive 

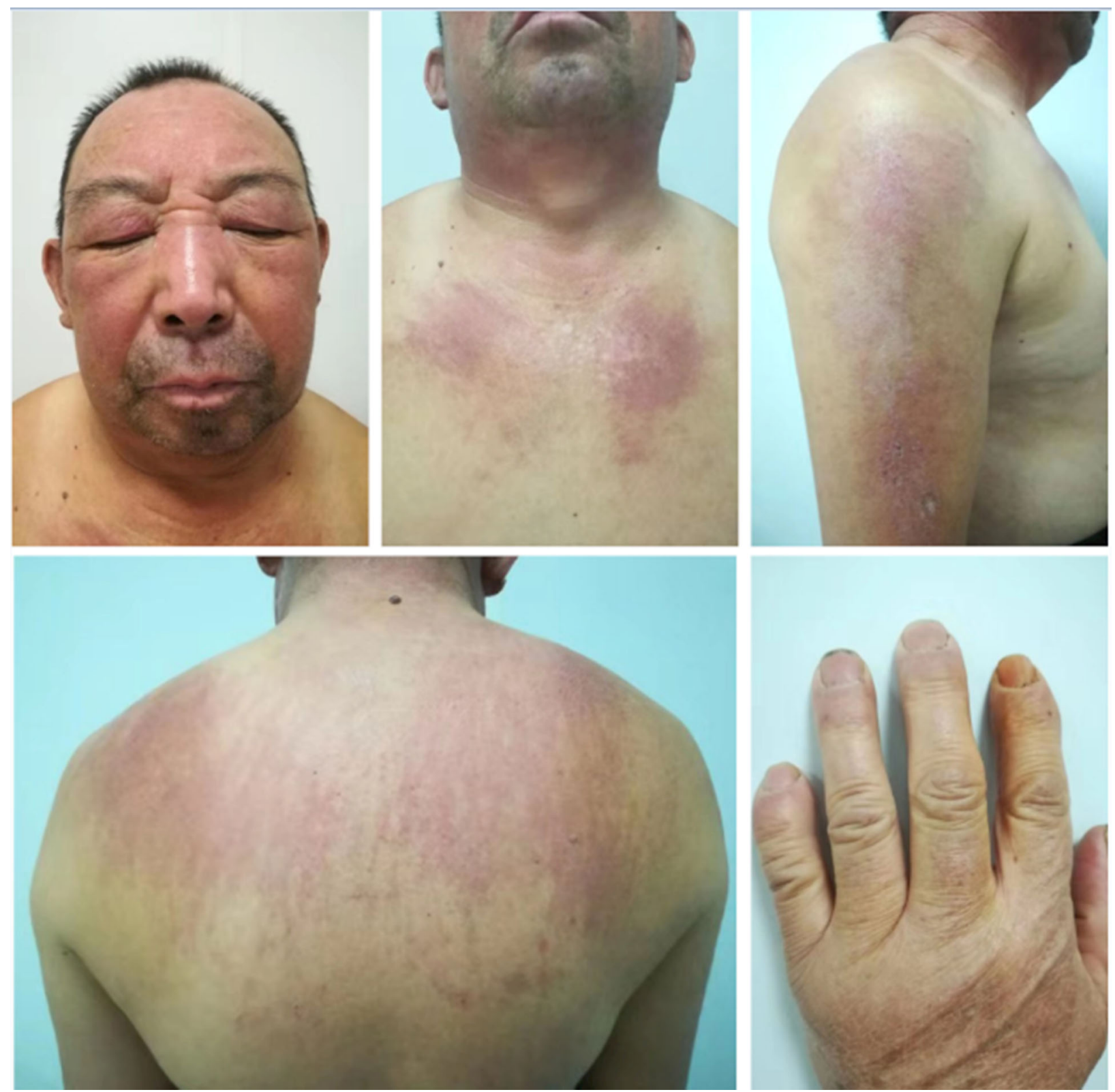

Fig. 1 Cutaneous manifestations of the patient showing heliotrope rash on the head, face, and chest, and purplish-red scaly maculopapules and poikiloderma on the scapular region, upper arm extension, and dorsal hands

with a titer of $1: 100$, with a speckled pattern; there were no additional detectable autoantibodies. Electroneuromyography revealed myogenic involvement of the deltoid muscle. The patient was diagnosed with dermatomyositis. The skin rash and muscle weakness were relieved after oral administration of prednisone $1 \mathrm{mg} / \mathrm{kg}$ per day and discontinuation of toripalimab. However, the symptoms reappeared and were aggravated soon after treatment with toripalimab was once again initiated, leading us to suggest that he stop toripalimab therapy. The symptoms were ultimately controlled by treatment with a once-daily dose of intravenous methylprednisolone $1 \mathrm{mg} / \mathrm{kg}$ for 7 days, followed by gradually tapering. During 
follow-up, computed tomography and ultrasonic examination revealed no obvious progression of the metastatic melanoma.

\section{DISCUSSION}

Dermatomyositis is an autoimmune disease characterized by proximal muscle weakness and typical skin manifestations. It can occur spontaneously or in association with neoplastic disorders, presenting as paraneoplastic dermatomyositis. Occasionally, it may also occur secondary to certain drugs, including lipid-lowering agents, penicillamine, hydroxynurea and, rarely, checkpoint inhibitors used in immunotherapy $[2,3]$. The patient in our case has metastatic melanoma. Paraneoplastic dermatomyositis was initially suspected. However, the onset and relapse of dermatomyositis soon after use and reuse of toripalimab and the relief of symptoms after toripalimab had been discontinued are highly suggestive of immunotherapy-induced dermatomyositis. The stable status of the metastatic melanoma of the patient during the period is also indicative of immunotherapy-induced dermatomyositis.

Checkpoint inhibitor immunotherapy is an effective treatment strategy for advanced malignancies, especially for patients whose previous standard therapy failed. Among these, cytotoxic T-lymphocyte antigen-4 (CTLA-4) inhibitors, such as ipilimumab, and PD-1 inhibitors, such as pembrolizumab and nivolumab, have shown remarkable effects on metastatic melanomas. Toripalimab is a new PD- 1 inhibitor and was approved for the treatment of advanced melanoma by China's National Medical Products Authority (NMPA) in December 2018 [1], subsequent to approval for pembrolizumab and nivolumab. Pembrolizumab is a humanized monoclonal IgG4- $\kappa$ antibody with high affinity for PD-1, nivolumab is a fully humanized IgG4 monoclonal antibody directed against the PD-1 receptor, and toripalimab is recombinant humanized monoclonal antibody that binds to PD- 1 and prevents PD- 1 from a binding with its ligands. In clinical trials, toripalimab has demonstrated a similar objective response rate (ORR) as pembrolizumab [4, 5].
PD-1 belongs to the CD28/B7 immunoglobulin superfamily. Since PD-1 inhibitors prevent the inhibition of T-cell activity by blocking selftolerance in a nonspecific fashion, they may lead to various irAEs, including cutaneous reactions, which might appear frequently. To our knowledge, this is the first case of dermatomyositis reported as an irAE induced by the new PD-1 inhibitor toripalimab. Our patient developed symptoms of dermatomyositis after two cycles of immunotherapy with toripalimab, similar to a case of dermatomyositis induced by nivolumab, and most patients develop symptoms within the first four cycles $[3,6]$. The early appearance of symptoms may help remind clinicians of the suspicion of irAES due to immunotherapy.

\section{CONCLUSIONS}

In conclusion, we report the first case of dermatomyositis caused by the new PD-1 inhibitor toripalimab in a patient with metastatic melanoma. The dermatomyositis was controlled rapidly by discontinuing toripalimab threapy and administering once-daily intravenous methylprednisolone $1 \mathrm{mg} / \mathrm{kg}$. This case should make clinicians aware of the potential for immunotherapy-induced dermatomyositis as an irAE. Early recognition and treatment may prevent progression and improve prognosis of this entity.

\section{ACKNOWLEDGEMENTS}

Funding. This study is funded by Natural Science Foundation of Liaoning Province (2019MS-094), which is being used to follow up the patient. No funding was received for the journal's rapid service fee.

Authorship. All named authors meet the International Committee of Medical Journal Editors (ICMJE) criteria for authorship for this article, take responsibility for the integrity of the work, and have given their approval for this version to be published. 
Authorship Contributions. Jingrong Lin wrote the report. Minmin Xue, Mingyang Gao, $\mathrm{Pu} \mathrm{Yu}$ and Shixin Han made the diagnosis and treated the patient.

Disclosures. Jingrong Lin, Minmin Xue, Mingyang Gao, $\mathrm{Pu} \mathrm{Yu}$, Shixin Hanhave nothing to disclose.

Compliance with Ethics Guidelines. Informed consent was obtained from the patient for publication of the article. We thank the patient for his written consent for using his pictures and data.

Data Availability. Data sharing is not applicable to this article as no datasets were generated or analyzed during the current study.

Open Access. This article is licensed under a Creative Commons Attribution-NonCommercial 4.0 International License, which permits any non-commercial use, sharing, adaptation, distribution and reproduction in any medium or format, as long as you give appropriate credit to the original author(s) and the source, provide a link to the Creative Commons licence, and indicate if changes were made. The images or other third party material in this article are included in the article's Creative Commons licence, unless indicated otherwise in a credit line to the material. If material is not included in the article's Creative Commons licence and your intended use is not permitted by statutory regulation or exceeds the permitted use, you will need to obtain permission directly from the copyright holder. To view a copy of this licence, visit http://creativecommons.org/licenses/by$\mathrm{nc} / 4.0 /$.

\section{REFERENCES}

1. Keam SJ. Toripalimab: first global approval. Drugs. 2019;79(5):573-8.

2. Bogdanov I, Kazandjieva J, Darlenski R, Tsankov N. Dermatomyositis: current concepts. Clin Dermatol. 2018;36(4):450-8.

3. Kosche C, Stout M, Sosman J, Lukas RV, Choi JN. Dermatomyositis in a patient undergoing nivolumab therapy for metastatic melanoma: a case report and review of the literature. Melanoma Res. 2019;30(3): 313-6.

4. Tang B, Yan X, Sheng X, et al. Safety and clinical activity with an anti-PD-1 antibody JS001 in advanced melanoma or urologic cancer patients. J Hematol Oncol. 2019;12(1):7.

5. Si L, Zhang X, Shu Y, et al. A phaseIb study of pembrolizumab as second-line therapy for Chinese patients with advanced or metastatic melanoma (KEYNOTE-151). Transl Oncol. 2019;12(6):828-35.

6. Sheik Ali S, Goddard AL, Luke JJ, et al. Drug-associated dermatomyyositis following ipilimumab therapy: a novel immune-mediated adverse event associated with cytotoxic T-lymphocyte antigen 4 blockade. JAMA Dermatol. 2015;151(2):195-9. 